\title{
SPOTTED
}

\section{Spotted around the web: Roundworm connectome, NRXN1 rats, MRI headphones}

BY JILL ADAMS, ANGIE VOYLES ASKHAM, PETER HESS, NIKO MCCARTY

6 AUGUST 2021 